\title{
Rethinking atomic packing and cluster formation in metallic liquids and glasses
}

\author{
LI QiKai ${ }^{1} \&$ LI Mo ${ }^{1,2 *}$ \\ ${ }^{1}$ Department of Mechanical Engineering, Tsinghua University, Beijing 100084, China; \\ ${ }^{2}$ School of Material Science and Engineering, Georgia Institute of Technology, Atlanta, GA 30332-0245, USA
}

Received August 7, 2011; accepted August 31, 2011

In traditional view, atomic packing is random in glasses made of metallic elements with non-directional interactions as the glass-forming liquid needs to be excited to remain in liquid state before being cooled sufficiently fast to a glass. Locally ordered packing however is possible if certain conditions are favorable, such as a strong bonding between elements, or low configuration energy of a cluster of atoms as suggested by Frank. In alloy systems made of different metallic elements, we show that Frank's criterion alone does not necessarily lead to certain specific local ordered packing or cluster formation such as icosahedral packing. In this context, we revisit the issue of atomic packing and cluster formation, and show that an alloy system with fairly random liquid configuration could be sufficient to produce a variety of noticeable locally ordered packing with low energy, albeit largely statistical in nature. Therefore, we emphasize the importance of the system parameters such as the atomic size, alloy concentration, and interaction potential in their collective contribution to local atomic packing.

metallic glass, short and medium range order, icosahedral order, glass formation

Citation: Li Q K, Li M. Rethinking atomic packing and cluster formation in metallic liquids and glasses. Chinese Sci Bull, 2011, 56: 3897-3901, doi: 10.1007/s11434- 011-4833-0

The question of whether or not a liquid should have a locally ordered structure has a significant bearing on many issues in materials science and condensed matter physics. To some extent, stability of a liquid depends on whether or not such an ordered structure exists, so deep undercooling could be achieved [1]. A glass-forming liquid with extended stability is a precursor for good glass forming ability as the cooling rate $q=\Delta T / \Delta t$ is determined by the temperature gap $\Delta T=T_{1}$ $-T_{\mathrm{g}}$ between the liquidus line $T_{1}$ and the glass transition temperature $T_{\mathrm{g}}$, and $\Delta t$ which is the time taken during cooling across the gap. A critical cooling rate is represented by the slowest $q$ for a system. Obviously, a system with the smallest $\Delta T$ would have the slowest cooling rate at a sufficiently long cooling time to avoid crystallization, thus more stable [2]. When local atomic packing with certain noncrystalline order is present, the liquid would have lower configuration energy and therefore a low $T_{1}$. So the liquid

\footnotetext{
*Corresponding author (email: mo.li@mse.gatech.edu)
}

becomes more stable and could be made relatively easily into a glass $[1,2]$.

One such locally ordered packing is icosahedral cluster made of 13 atoms. As proposed by Frank [1], by considering the pairwise interactomic interactions, i.e. the LennardJones (LJ) interaction, $\phi_{\mathrm{LJ}}(r)$, between atoms separated by a distance $r$, the potential energy of the central atom interacting with the 12 nearest neighbor atoms would be about $8.4 \%$ lower if they are packed as an icosahedron than that if they are packed as the face-centered-cubic (fcc) structure. An icosahedron packing requires that all 12 neighboring atoms are located at the vertex points of 3 perpendicular rectangles with the golden ratio. In contrast, an fcc packing has all 12 neighboring atoms sitting at the vertices of 3 perpendicular squares. As a result, the ratio between the nearest neighbor distances in an icosahedron is shorter than that in an fcc by $\sqrt{1+[(1+\sqrt{5}) / 2]^{2}} / 2 \approx 0.95$, which thus results in lower configuration energy. Correspondingly, the lattice 
energy with icosahedron clusters is about $40 \%$ lower. Therefore, this low energy atomic packing is the preferred local structure and would be expected to permeate a liquid [1].

Frank's idea has attracted great attention in the past 50 years, especially in the community of metallic glass. Briant and Burton [3] were among the first to propose that amorphous metals could be composed of high density of these low energy clusters. For this reason, they called icosahedron the "structural unit" of amorphous metals. Using various model clusters of different number of atoms, they showed that the scattering function of the 13-atom icosahedral cluster is much closer to some of the known experimental diffraction functions of metallic glasses, which made them to conclude that amorphous metals should be composed of these low energy structural units. Glass transition, on the other hand, would involve the development of these structural units in undercooled liquid with random orientation [4,5]. Extensive molecular dynamics simulations were performed to measure and characterize the icosahedral clusters, albeit most of which are in one-component Lennard-Jones (LJ) systems [6,7]. The anticipated icosahedral packing was indeed found in these systems; and moreover, the glass transition was found to occur in tandem with the increasing icosahedral cluster formation.

This simple and appealing idea has found its way into alloy systems composed of different metallic elements. Different from the one component systems with equal sized atoms, in mixed systems the icosahedral cluster would be ideal if the central atom has the atomic radius about 5\% smaller than that of the 12 surrounding neighbor atoms, so the neighboring atoms would be in direct contact with each other to form a tightly packed icosahedron. As shown by Jonsson and Andersen [7], about 10\% increase in the representative icosahedral motif was seen in a binary Lennard-Jones liquid where the atoms have about $10 \%$ atomic size difference, upon cooled to the glass transition. In a model Mg-Ca binary liquid, a majority of the local packing with five-fold symmetry was also reported with cooling [8]. More dramatic presence of the icosahedral packing has been reported recently in some of the bulk metallic glass systems, most noticeably those made of $\mathrm{Zr}$ and $\mathrm{Cu}$ [9-13]. The finding leads to the impression that the good glass-forming liquids are those with the excessive amount of locally ordered icosahedral packing or clusters with lower configuration energy. Further extension of this conclusion led to the suggestion that many material properties are affected significantly by the local atomic packing, which includes the glass formability, brittle vs. ductile behavior in the mechanical response, lower viscosity and diffusivity, etc. in bulk glass forming systems [14-16].

In this letter, using available data and simple analysis we shall arrive at the following two general conclusions that are not necessarily in agreement with the current thinking, that is, (1) in alloy systems the atomic packing, especially the icosahedral order is not governed necessarily by Frank's energy criterion, and (2) rather the formation of the clusters or local order, to a great extent, is a consequence of freezing a relatively random or stochastic liquid with metastable configurations. The configurations are dictated largely by the system parameters such as the atomic size and interatomic interactions, and the cooling condition. These conclusions may be reflected more obviously in computer simulations when insufficient relaxation time is given.

First, let us consider the typical binary system made of $\mathrm{Zr}$ and $\mathrm{Cu}$ in which a few best glass forming liquids were discovered [17]. The interatomic potentials among the alloy elements are shown in Figure 1. Since the many-body effects are considered in the interatomic interactions [18,19], the potentials in Figure 1 are the effective pair potential. As compared with pure elements, alloy systems have two distinct features. One is that the elements have different effective atomic radii and the second is that the interaction or bonding energy is different among different elements. Both of these features can be seen in the effective potentials in Figure 1. The atomic radius of $\mathrm{Cu}$ and $\mathrm{Zr}$ is about 135 and $175 \mathrm{pm}$ respectively [20,21] which are quite close to the distance at the minimum of the $\mathrm{Cu}-\mathrm{Cu}$ and $\mathrm{Zr}-\mathrm{Zr}$ interatomic potentials. The atomic size ratio $\eta$ between $\mathrm{Cu}$ and $\mathrm{Zr}$ is 0.77. From Figure 1, we can also see that the bonding energy at the first neighbor distance between $\mathrm{Cu}-\mathrm{Cu}$ atoms is about twice as small as that of $\mathrm{Zr}-\mathrm{Zr}$, while the $\mathrm{Cu}-\mathrm{Zr}$ bonding energy is in between. With these parameters in mind, the atomic size and the first neighbor bonding energy, we now can proceed with our analysis.

Then, let us consider the isolated cluster composed of $M+1$ atoms where $M$ is the number of neighboring atoms around the central atom. In the treatment of local atomic packing in condensed phase such as liquid and solid, we can safely assume that the hard-core repulsion of the interatomic interaction plays a primary role and the detailed bonding energy plays a secondary role. Therefore, we can predict the number of neighbor atoms surrounding a central atom, or

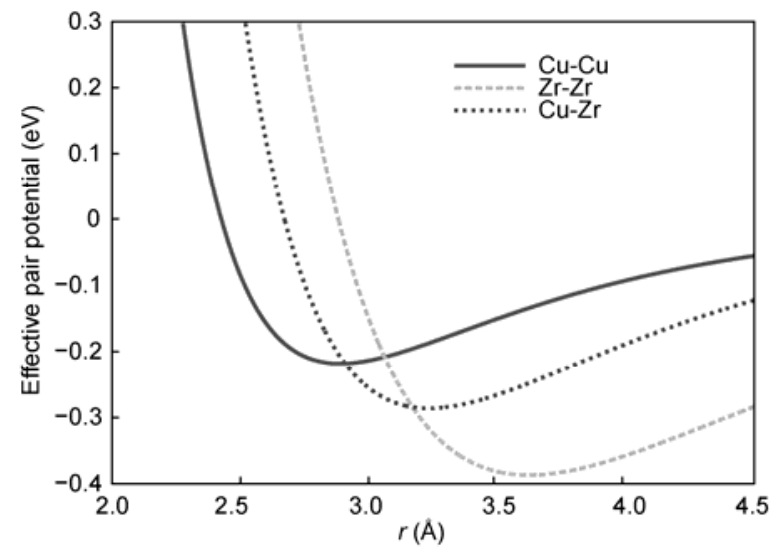

Figure 1 The effective pair interactions among $\mathrm{Zr}-\mathrm{Zr}$, $\mathrm{Zr}-\mathrm{Cu}$, and $\mathrm{Cu}-\mathrm{Cu}$ for the $\mathrm{Cu}-\mathrm{Zr}$ system. 
the coordination number of a $M+1$ cluster. If a central atom with radius $r_{\mathrm{A}}$ is surrounded by atoms with the radius $r_{\mathrm{B}}$, to fully surround the central atoms (with no open space large enough to fit another neighboring atom in), there should be $M=4 \pi(1-\sqrt{3} / 2) /(1-\sqrt{1+2 \eta} / 1+\eta)$ neighboring atoms $[22,23]$, plus and minus a few number of atoms from statistical fluctuation due to temperature and finite compressibility of the neighboring atoms. Here $\eta=r_{\mathrm{B}} / r_{\mathrm{A}}$ is the atomic size ratio.

From the atomic size ratio between the neighbor atoms and the central atom, we can predict roughly the coordination number $M$. Therefore, if the central atom is the larger sized $\mathrm{Zr}$ atom, the minimum number of its neighbors would be 12 if all the neighbors are $\mathrm{Zr}$, and the maximum is about 17 if all the neighbors are small sized $\mathrm{Cu}$ atom. Similarly, if the central atom is the small sized $\mathrm{Cu}$ atom, the minimum number of the neighbors would be 10 if all of them are the large $\mathrm{Zr}$ atom and the maximum is 12 if all its neighbors are small sized $\mathrm{Cu}$ atoms. Therefore, we can immediately see that the number of neighbors $M$, or the cluster coordination number would appear as a bimodal distribution with one peak centered on $\mathrm{Cu}$ atom and another around $\mathrm{Zr}$ atom. The distribution of the coordination number, or cluster size is bound by the limits we just predicted. Of course, the above prediction based on hard-core model would be relaxed in reality when the system is allowed to have density fluctuation or at certain temperature. For example, the upper limit of the coordination number for $\mathrm{Cu}$ may range from 6 to 13 (or 14) and for $\mathrm{Zr}$, it is 8 to 19 (or 20).

Another unique feature of an alloy in terms of atomic packing is the mixing of different alloy elements in the neighbor shells. As different elements start to mix, the cluster size distributions would vary but the variation would be within the limits we gave above. Therefore, one may see a bit wider distribution of the neighbor number $M$ in experimental or computer simulated samples, nevertheless the bimodal feature should remain.

Such a simple outcome tells us that in $\mathrm{Cu}-\mathrm{Zr}$ system the cluster size follows a bimodal distribution with two peaks determined by alloy's different atomic sizes. In contrast, one-component system should have only one peak. In the case of the one-component LJ system, the peak is centered at the mean of about 10 in liquid state and 12 in crystalline state (see Figure 9 in [24]). Therefore, the larger the atomic size difference is in a binary system, the larger the peak separation is. However, as the number of alloy component increases, the distinction of the cluster size may become less obvious if the atomic sizes of the elements are distributed within a narrow range.

From these predictions, we can see that icosahedral order characterized by the coordination number around $M=12$ and fivefold symmetry may not be necessarily the dominated one in total number, or not even be present as envisioned before [1-8] in alloy systems if the atomic size ratio is not in certain favorable range. One such example is the metalmetalloid systems with small elements such as $\mathrm{P}, \mathrm{B}$ or $\mathrm{C}$ [25]. It is interesting however to notice that the atomic size ratio between most metallic elements in all metal-metal metallic glasses are within 0.7-1.3 [2], so the icosahedral packing is certainly a common occurrence, although may not necessarily be the dominant one. The analysis shown above indicates that the clusters in an alloy such as $\mathrm{Cu}-\mathrm{Zr}$ would have a broad range in cluster size distribution, from $M=6$ to 20 as dictated predominantly by the geometric factor of the atomic sizes or repulsive interatomic interactions. One should expect that the local packing is optimized to achieve high or most efficient packing density [22,23] due to the sufficient thermal agitation in liquid state.

The next question is whether or not there is a preferred cluster such as the icosahedral cluster that has an overwhelming presence as Briant and Burton proposed [3]. Our first answer to this question is that the relative amount of these clusters depends on not only the bonding energy of the cluster, as Frank suggested, but also the concentration of the alloy elements. For a binary system with relative concentration of one type of atom being $x$, the mean number of neighbors of one type of atom around the other type of atom scales as $x(1-x)$. For $\mathrm{Cu}-\mathrm{Zr}$ system, if the concentration of $\mathrm{Cu}$ is small (less than $50 \%$ ), the relative amount of small clusters with the nearest neighbor less than 14 is small, including that of the icosahedra because of the relative scarcity of $\mathrm{Cu}$ that could serve as the center atom for the cluster. For the same reason, if the $\mathrm{Zr}$ concentration is small, the large size cluster with the nearest neighbors larger than 14 is small. Therefore, we would expect a smooth change of the relative amount of the different types of clusters as the alloy concentration changes from the $\mathrm{Cu}$ poor to the $\mathrm{Cu}$ rich region of the phase diagram. The packing density of $\mathrm{Cu}-\mathrm{Zr}$ binary system in three-dimensions may very much like what appears Ref. [26], at least qualitatively with the maximum packing density at around $x=0.5-0.7$. Note that the cluster distribution obtained this way is from random packing. Whether or not there should be an abnormal increase of packing density with certain type of clusters made of low configuration energy that correspond to the best glass forming range, however, remains to be resolved [27].

The second answer is that the amount of certain clusters depends on their relative configuration energy, as in Frank's argument. While the "hard-core repulsion" dictates the limits of atomic packing as shown above, let us now look at the energetic of these clusters. Since the bonding energy of the nearest neighbors is governed by the potential energy shown in Figure 1, we can estimate the energy of the clusters predicted above. For the $\mathrm{Cu}$-centered clusters, for $M=9$, the potential energy is about $-2.7 \mathrm{eV}$ per atom as most of its neighbors are $\mathrm{Zr}$ atoms if we consider the nearest neighbor interactions. On the other extreme, if all neighbors are $\mathrm{Cu}$, one has $M=12$ neighbors, presumably forming an icosahedrons, the bonding energy for the center $\mathrm{Cu}$ atom is about 
$-2.4 \mathrm{eV}$. As we gradually dilute the neighbors with increasing number of $\mathrm{Cu}$, the potential energy is going to change from the lower bound at about $-2.7 \mathrm{eV}$ to the upper bound at about $-2.4 \mathrm{eV}$. So the bonding energy of the other $\mathrm{Cu}$-centered clusters should be between -2.7 and $-2.4 \mathrm{eV}$ as more $\mathrm{Cu}$ mixes with $\mathrm{Zr}$. Of course, at any of the limits, certain deviations may occur in both the total number of the neighbors or the relative concentration of $\mathrm{Cu}$ and $\mathrm{Zr}$ in the first neighbor shell. The latter leads to the distorted icosahedrons for the clusters around $M=12$.

For the $\mathrm{Zr}$-centered clusters, the energy is the lowest for $M=12$ neighbors made of all $\mathrm{Zr}$, that is, $-4.8 \mathrm{eV}$. As more small-sized $\mathrm{Cu}$ mixed into the first neighbor shell around $\mathrm{Zr}$, the energy begins to increase (while the number of the first neighbors increases, too), as indicated by the higher $\mathrm{Zr}-\mathrm{Cu}$ interaction (Figure 1). Such trend continues in clusters with $M>12$. Since the bonding energy of $\mathrm{Zr}-\mathrm{Zr}$ and $\mathrm{Zr}-\mathrm{Cu}$ is lower than that of $\mathrm{Cu}-\mathrm{Cu}$, we expect the bonding energy of the $\mathrm{Zr}$-centered clusters have lower configuration energy than the predominantly $\mathrm{Cu}$ rich clusters, including the icosahedral clusters.

From the above analysis, we can see that the icosahedra, most of which are Cu-centered, should not be the energetically most favorable ones. Our recent numerical work confirms this conclusion (unpublished results). In fact, the conclusion is not surprising at all. In one component systems, Frank's energy criterion for cluster formation is specific as he compares the icosahedrons with the reference state of the fcc or hcp packing. In alloy systems, because of different bonding energy and atomic size and the possibilities of a large number of topological and chemical arrangements of the atoms, Frank's energy criterion becomes ambiguous to apply. For example, for the $M=12$ cluster in an alloy system, it is no longer obvious to us how to compare the energy of an existing icosahedral cluster made of a mixture of $\mathrm{Cu}$ and $\mathrm{Zr}$ atoms with that of a "would-be" reference cluster with $M=12$ whose state or structure is either the complex eutectic crystal mixture, or in general unknown if it is a metastable phase. Another alternative is to compare the energies of different clusters. But as we saw in the above analysis, such comparison does not work either, as there are many clusters that have lower bonding energy than the icosahedra.

The above cases were for isolated clusters, that is, the interactions of the neighbors with other atoms outside of the cluster are neglected. One expects that the outcome may be different once those interactions are included. Since a large portion of the bonding energy (up to $\sim 40 \%$ ) is usually contributed from the first neighbor interactions, including the interactions from the remote neighbors may not change the scenario qualitatively. Indeed, as shown in recent work $[28,29]$ including these interactions explicitly only adds minor changes in the cluster energy.

Now we are ready to discuss the next question: How the clusters form. One scenario is directly from Frank's energy criterion, that is, clusters form as driven by the configura- tion energy difference: The lower the cluster configuration energy, the more populated the types of the clusters. This proposal must involve certain types of growth of the low energy configuration clusters. But as we show below, the "growth" process is quite limited by many factors. The other possible mechanism, which we propose here, is the result of kinetic freezing of the default random configurations in the liquid state with certain degrees of local optimization of atomic packing $[22,23]$. The major difference between these two mechanisms lies in that the latter does not assume a (significant) growth process of the clusters with lower configuration energy from those of higher configuration energies. For example, if the growth occurs, the lower energy configuration of the $\mathrm{Zr}$-centered clusters would be populated, rather than the $\mathrm{Cu}$-centered icosahedrons. In order to do so, significant diffusion is required so the elements in clusters with higher configuration energy need to migrate to form the low energy configuration clusters. The growth is hindered by the high activation barrier of rearranging both $\mathrm{Cu}$ and $\mathrm{Zr}$ atoms so the elements can diffuse from the $\mathrm{Cu}$-rich environment (with high energy) to the $\mathrm{Cu}$-poor environment (with low energy). Besides, it is impossible to have certain number of the low energy clusters as it is restricted by the overall composition of the system. Under these constraints, the glass-forming liquids would take the configurations primarily from the liquid in the excited state with nearly random configuration and some possible locally optimized packing. In this picture, the clusters are largely dictated by the packing rules set by the atomic size, overall composition, and bonding energy in the short-range region.

In summary, we reexamined the Frank's energy criterion for local atomic packing in alloy systems, using $\mathrm{Cu}-\mathrm{Zr}$ binary system as an example. We showed that straightforward extension of Frank's criterion from single component system to alloy system is invalid, as the reference state to compare with remains ambiguous. A broad spectrum of local packing exists that is dictated largely by the default liquid configuration maintained at the high temperature excited state. In particular, we showed that the clusters with icosahedral symmetry do not have the lowest configuration energy among the clusters available. The atomic packing is not necessarily dominated by a single type of clusters, or even by these with lowest configuration energy. The atomic configurations are however optimized locally by the rules set by the atomic size, overall composition, and bonding energy.

This work was partially supported by the Tsinghua University and the National Thousand Talents Program of China.

Frank F C. Proc Roy Soc London A, 1952, 215: 43-46

Jalali P, Li M. Phys Rev B, 2005, 71: 013450

Briant C L, Burton J J. Phys Stat Sol B, 1978, 85: 393-402

Nelson D R. Phys Rev B, 1983, 28: 5515-5535

Stephens P W, Goldman D R. Phys Rev Lett, 1986, 56: 1168-1171

Steinhardt P J, Nelson D R, Rohchetti M. Phys Rev B, 1983, 28: 
784-805

7 Jonsson H, Andersen H C. Phys Rev Lett, 1988, 60: 2295-2298

8 Qi D W, Wang S. Phys Rev B, 1991, 44: 884-887

9 Saida J, Kasai M, Matsubara E, et al. Ann Chim (Paris)—Sci Mater, 2002, 27: 77-89

10 Louzguine D V, Fukuhara M, Inoue A. Acta Mater, 2007, 55: 1009

11 Cheng Y Q, Sheng W H, Ma E. Phys Rev B, 2008, 78: 014207

12 Senkov O N, Miracle D B. J Non-Cryst Solids, 2003, 317: 34

13 Lee M, Lee C M, Lee K R, et al. Acta Mater, 2011, 59: 159

14 Lekka Ch E, Ibenskas A, Yavari A R, et al. Appl Phys Lett, 2007, 91: 214103

15 Kelton K F, Lee G W, Gangopadhyay A K, et al. Phys Rev Lett, 2003, 90: 195504

16 Wessels V, Gangopadhyay A K, Sahu K K, et al. Phys Rev B, 2011, 83: 094116
17 Xu D H, Lohwongwatana B, Duan G, et al. Acta Mater, 2004, 52: 2621

18 Duan G, Xu D H, Zhang Q, et al. Phys Rev B, 2005, 71: 224208

19 Cleri F, Rosato V. Phys Rev B, 1993, 48: 22

20 Buch A. Short Handbook of Metal Elements Properties and Elastic Properties of Pure Metals. Warsaw: Krzysztof Biesaga, 2005

21 Slater J C. J Chem Phys, 1964, 41: 3199

22 Egami T, Waseda Y. J Non-Crystal Solids, 1984, 64: 113

23 Jalali P, Li M. J Chem Phys, 2004, 120: 1138

24 Bai X M, Li M. Phys Rev B, 2008, 77: 134109

25 Boudreaux D S, Gregor J M. J Appl Phys, 1977, 48: 152

26 Jalali P, Li M. Physica A, 2007, 381: 230

27 Li Y, Guo Q, Kalb J A, et al. Science, 2008, 322: 1816-1819

28 Mossa S, Tarjus G. J Chem Phys, 2002, 119: 8069

29 Yao Y X, Wang C Z, Ho K M. Phys Rev B, 2007, 76: 174209

Open Access This article is distributed under the terms of the Creative Commons Attribution License which permits any use, distribution, and reproduction in any medium, provided the original author(s) and source are credited. 